\title{
Predator-Prey Relationships System
}

\author{
Taleb A.S. Obaid, PhD \\ Department of Computer Information System, \\ College Of Computer Science and Information \\ Technology, \\ Basrah University, \\ Iraq.
}

\begin{abstract}
Most of the ecological systems have the elements to produce divisions and dynamics behavior, and food chains are ecosystems with familiar structure. Modeling efforts of the dynamics of food chains which are initiated long ago confirm that food chains have very rich dynamics. This work focused on applying biological mathematical model to analyzing predation or competition relationships in the natural environment between predators and preys. We are interesting to consider two species of animals; interdependence might arise because one species (the "prey") serves as a food source for the other species (the "predator"). Models of this type are thus called predator-prey models. Initially, we exercised the mathematical model of one prey and one predator. Later on, we considered very excited model that dealing with one predator and two preys. The populations of the prey and predator will be modeled by two differential equations for the early case and with three differential equations for a later model. The Matlab command ode45 can be used to solve such systems of differential equations.
\end{abstract}

\section{Keywords}

Predation, ecosystem, differential equation, Lotka-Volterra model.

\section{INTRODUCTION}

Mathematical population models have been used to study the dynamics of prey predator systems since Lotka (1925) [1] and Volterra (1927) [2]. They proposed the simple model of preypredator interactions relationship called the Lotka-Volterra model. The predation relationship interacts in a relatively isolated manner compared to other species. So, their populations fluctuated in a regular cycle due to lack of significant external variables on the relationship. Competition is always rooted in the issue of organisms fighting for the same booty, limited resource, in an ecosystem. Interspecific competition is defined as two or more different species competing for the same resource, and intraspecific competition is between members of the same species.

Predators and prey can influence one another's evolution, so each of them trying to gain the competitive advantages. Traits that enhance a predator's ability to find and capture prey will be selected for the predator to keep survive. While trait that enhance the prey's ability to avoid being eaten (victim) will be selected for in the prey. The "goals" of these traits are not compatible, and it is the interaction of these selective pressures that influences the dynamics of the predator and prey populations. Predicting the outcome of species interactions is also of interest to biologists trying to understand how communities are structured and sustained [3].

\author{
Alaa Khalaf Hamoud \\ Department of Computer Information System, \\ College Of Computer Science and Information \\ Technology, \\ Basrah University, \\ Iraq.
}

We already used systems of linear ordinary differential equations to define and analyze these population fluctuations, mathematically, upon several minimizing assumptions in order to exclude incalculable variables. The mathematical model "Lotka-Volterra" can be analyzed analytically or using computer simulation to determine some practical information to the reality of the relationship.

Green (2004) [4], studied a model on two preys-one predator system in which the prey were non competing and predation followed the density gradient of the prey. It was discovered that when the predator divides its time between the two preys depending on their comparative density, the predator stabilized the system.

Vlastmil and Eisner (2006) [5], studied a one consumer (predator)-two resource (preys) population dynamics system in which the resource was spatially distributed between two patches. The study showed that when resources grow exponentially, handling times are zero and apparent competition always leads to extinction of the weaker resource.

\section{PREDATOR-PREY INTERACTION}

We will implement more than two population's models where they depend on each other. One population could be the predator and the other the prey. The populations will be modeled by two ordinary differential equations. The second model could be one predator and two preys. The second model populations will be treated by three differential equations. In order to solve the mathematical model, (Matlab command ode45) used to solve such systems of differential equations, [6].

Differential equations are a mathematical means to describing the behavior of the natural world relations involving rates at which things occur. These relations become equations and the rates become derivate. So, any equations containing derivatives are differential equations.

\section{ONE PREDATOR AND ONE PREY}

Predation is the species interaction when one species, the predator, eats another species, the prey, as a source of food. The simplest and earliest mathematical model that describes such interaction is the Lotka-Volterra model, which is represented as:

$$
\begin{aligned}
& \frac{d x}{d t}=-\left(d+e_{1} y\right) x \text {, and } x(0)=x_{0} \\
& \frac{d y}{d t}=\left(k\left((M-y)-c_{1} x\right) y \text {, and } y(0)=y_{0}\right.
\end{aligned}
$$

Where $\mathrm{x}(\mathrm{t})$ represent the predator population and $\mathrm{y}(\mathrm{t})$ represents the prey population. The prey population will now have a logistic model when there are no predators. This means 
the birth and death rate for the prey population will vary and be set equal to $\mathrm{k}(\mathrm{M}-\mathrm{y})$.

- The parameter $\mathrm{k}, \mathrm{M}$ and $\mathrm{cl}$ must determine carefully [7-9].

- This model makes several critical assumptions which may be unrealistic in most real situations in nature. Specifically, the model assumes:

- The prey species has an unlimited food supply; the prey population grows exponentially in absence of predators.

- There is no other threat to prey other than one specific predator.

- The predator is completely dependent on the prey as the only food source. It will die in the absence of the specific prey instead of resorting to another food source.

\section{ONE PREDATOR AND TWO PREYS}

In the second case, we consider one predator and two preys. Let the second prey population be given by $z(t)$. Since the second prey population is also a prey, the birth rate minus the death rate of the second prey must be similar to the predator, so that birth rate minus death rate equals $\mathrm{k} 2(\mathrm{M} 2-\mathrm{z})-\mathrm{c} 2 \mathrm{x}$. The birth rate minus the death rate for the predator population will increase to $-\mathrm{d}+\mathrm{e} 1 \mathrm{y}+\mathrm{e} 2 \mathrm{z}$. Therefore, the new system of differential equations will have three equations:

$$
\begin{aligned}
& \frac{d x}{d t}=\left(-d+e_{1} y+e_{2} z\right) x \text {, and } x(0) x_{0} \text {, for predator } \\
& \frac{d y}{d t}=\left(k_{1}\left(M_{1}-y\right)-c_{1} x\right) y \text { and } y(0) y_{0} \text {, for first prey } \\
& \frac{d z}{d t}=\left(k_{2}\left(M_{2}-z\right)-c_{2} x\right) z \text { and } z(0) z_{0} \text {, for secondprey }(5)
\end{aligned}
$$

We will use the Matlab command ode 45 (ordinary differential
equations fourth and fifth order Runge-Kutta method) to solve systems of differential equation.

\section{NUMERICAL SIMULATION}

Analytical studies always remain incomplete without numerical verification of the results. Here, we present numerical simulation to illustrate the results obtained in previous sections. The numerical simulation was implemented in MATLAB R2008a.

In the yprf $2 . m$ file the $x(t)$, predator, is associated with the symbol $\mathrm{y}(1)$, and $\mathrm{y}(\mathrm{t})$, prey, is associated with the symbol $\mathrm{y}(2)$, as given in equations 1 an 2 . One can plot a solution which corresponds to the oscillatory nature of the population of the two species. This solution is in a state of dynamic equilibrium. At any given time in this phase plane, the system is in a limit cycle and lies somewhere on the inside of these elliptical solutions. There is no particular requirement on the system to begin within a limit cycle and thus in a stable solution, however, it will always reach one eventually.

function yprf2 =yprf2(t,y)

$\operatorname{yprf} 2(1)=-.5 * \mathrm{y}(1)+.01 * \mathrm{y}(1) * \mathrm{y}(2)$

$\operatorname{yprf} 2(2)=.0005 *(1000-\mathrm{y}(2)) * \mathrm{y}(2)-.01 * \mathrm{y}(1) * \mathrm{y}(2)$

yprf2 = [yprf2(1) yprf2(2)]';

The parameters values of the mathematical model eq (1) and (2):

The initial time $\mathrm{t} 0=0$, final time $\mathrm{tf}=50$; the population of prey and predator are yo $=[80100]$; call the matlab function "ode45" and then plot predator y(1) and prey y(2) population.

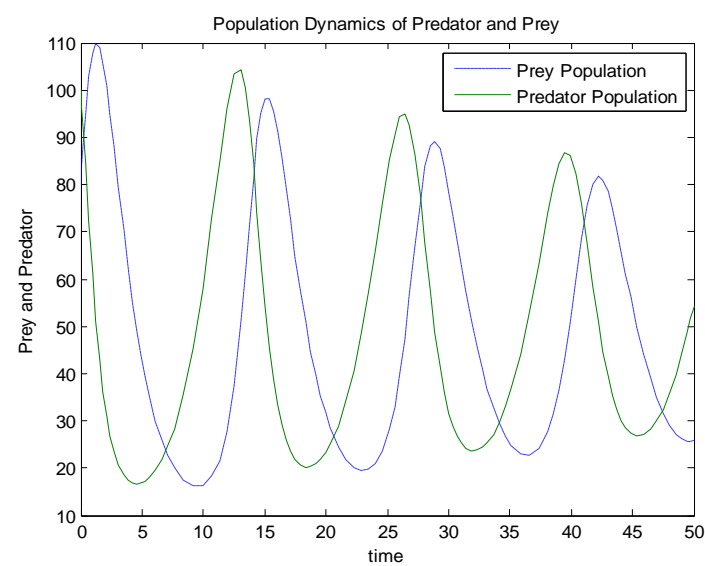

Figure 1: Simple Predator Prey Model

The phase plane plot compares the population of predators to the population of prey, and is not dependent on time.

Suppose there are two species of animals, a prey and a predator. If the initial conditions are 100 preys and 80 predators, one can plot the progression of the two species over time. The choice of time interval is arbitrary.

The parameters are: to $=0 ; \mathrm{tf}=50 ; \mathrm{yo}=[80100]$; and plot the predator and prey population against time to get the following figure.

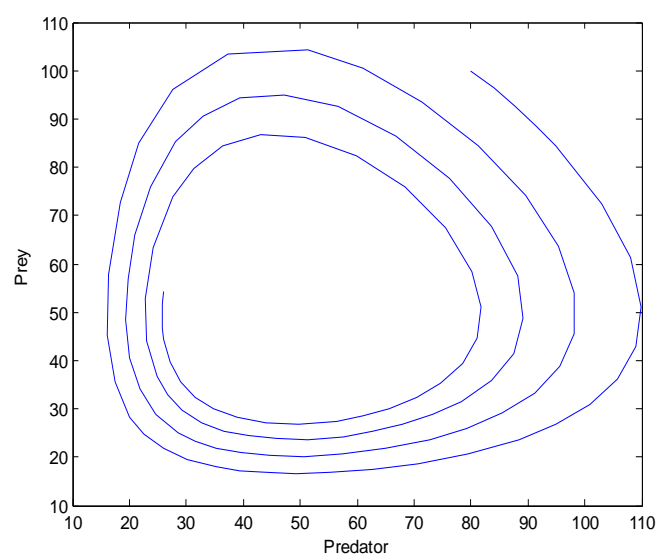

Figure 2: Predator Prey Phase Plots

It is easy to see from this graph that a large enough increase in the number of predators leads to a decrease in the number of prey. This is logical from a biological standpoint, since a larger population of predators leads to increased interactions between predators and prey, and therefore increased prey death.

In the following calculations we have to solve the one predator and two prey's model. In the yprf3.m file the $\mathrm{x}(\mathrm{t})$, predator, is associated with the symbol $\mathrm{y}(1)$; and $\mathrm{y}(\mathrm{t})$ and $\mathrm{z}(\mathrm{t})$, is the first prey and second prey, respectively; is associated with the symbol $\mathrm{y}(2)$ and $\mathrm{y}(3)$ as given in equations 3,4 , and 5 .

function yprft3 =yprft3(t,y)

$\operatorname{yprft} 3(1)=-.5 * \mathrm{y}(1)+.01 * \mathrm{y}(1) * \mathrm{y}(2)+.02 * \mathrm{y}(1) * \mathrm{y}(3) ;$

$\operatorname{yprft} 3(2)=.01 *(100-\mathrm{y}(2)) * \mathrm{y}(2)-.01 * \mathrm{y}(1) * \mathrm{y}(2)$;

$\operatorname{yprft3(3)}=.04 *(80-\mathrm{y}(3)) * \mathrm{y}(3)-.03 * \mathrm{y}(1) * \mathrm{y}(3$ 5 $)$ 
yprft3 = [yprft3(1) yprft3(2) yprft3(3)]';

The parameters of second mathematical model equations (3-5) as:

to $=0 ; \mathrm{tf}=20$; the initial populations of the predators, first preys, and second preys are yo $=[1009080]$;

We plot the population of predator and both preys against the time as:

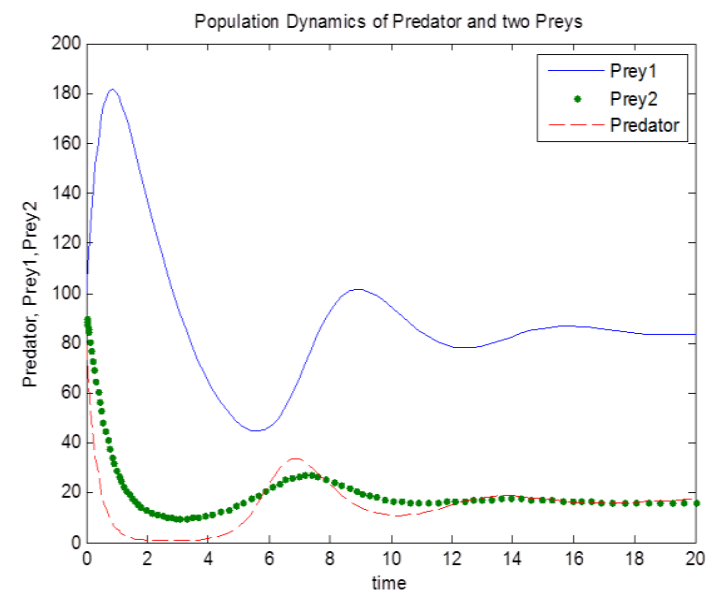

Figure 3: Population Dynamics of Predator and Two Preys

\section{CONCLUSION}

The most interesting dynamics in the biological world have to do with interactions between species. Mathematical models which incorporate these interactions are required if we hope to simulate these dynamics. This work is concerned with LotkaVolterra systems with constant terms. Two mathematical models were examined, a one-prey one predator and onepredator two preys, with various parameters. The predator and prey populations seem to cycle endlessly without settling down quickly
The dynamical behavior is found to be very sensitive to parameter values as well as the parameters of the practical life. This predation relationship is quite special, because these species interact in a relatively isolated manner compared to others, meaning their populations fluctuated in a regular cycle due to lack of significant external variables on the relationship. These population fluctuations can be defined and analyzed mathematically using systems of linear ordinary differential equations, built of course upon several minimizing assumptions in order to exclude incalculable variables.

\section{REFERENCES}

[1]. Lotka, A. J. (1925). Elements of physical biology. Williams and Wilkins. Baltimore, Maryland, USA.

[2]. Volterra, V. (1927). Variations and fluctuations in the numbers of coexisting animal species. The Golden age of theoretical Ecology; (1933- 1940). Lecture notes in Biomathematics, 22(1): 65-273, springer-Verlag, Berlin, Heidelberg, New York.

[3]. Guay M., "Dynamical systems and ecological modeling", Maryland Mathematical Modeling Contest, October 9th, 2014.

[4]. Green, E. (2004). The eff ects of a smart predator in a one predator-two prey system. University published. (http/green e/University of Chicago).

[5]. Vlastmil, K. and Eisner, J. (2006). The eff ect of Holling Type II functional response on apparent competition. Theoretical Population Biology, 70: 421-430.

[6]. Peterson J. K., "Predator Prey Models in MatLab", Clemson University, November 7, 2013

[7]. Wiklund K, Lundin J, Olsson P., V'agberg D, " Modeling and Simulation", Ume a University 2014

[8]. Von Arb R., "Predator Prey Models in Competitive Corporations", Olivet Nazarene University, 2013.

[9]. Knadler C. "Models Of A Predator-Prey Relationship In A Closed Habitat" Proceedings Simulation Conference, 2008. 